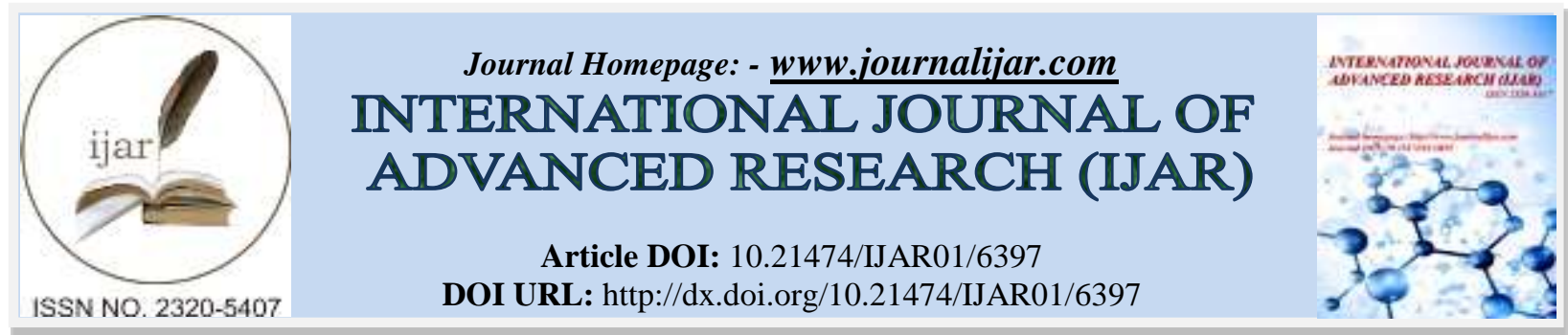

RESEARCH ARTICLE

\title{
LA PHILOSOPHIE DE LA POLITIQUE CHEZ MARCEL PAGNOL.
}

Dr. Nidhi Sharma.

\section{Manuscript Info}

Manuscript History

Received: 24 November 2017

Final Accepted: 26 December 2017

Published: January 2018

Key words:-

La société, voyez-vous, monsieur, si elle continue, elle tuera les justes.

\begin{abstract}
Un politicien est un homme impliqué dans l'activité politique. La plupart des politiciens font une preuve d'habileté dans leur vie politique. Dans cet article, nous allons discuter comment Marcel Pagnol s'amuse à se moquer des politiciens. Marcel Pagnol aborde de nombreuses questions politiques. Il nous montre bien comment ils tournent le dos aux problèmes du public. Les politiciens font une raillerie de la démocratie. Une fois la situation politique d'un ministre est solidement établie, il oublie ses devoirs et reste indifférent aux aspirations des gens. Le drame de la possession du pouvoir occupe presque toute la vie d'un politicien. La gloire lui monte à la tête. Cet article va aussi démontrer la manière dans laquelle Pagnol présente l'insouciance des politiciens vers le public. Un politicien n'est qu'un égoïste, un dur, un rétréci qui bafoue les valeurs au nom desquelles il a été élu. Pagnol nous suggère que la structure du système politique n'est pas sans rappeler les crimes, la corruption, l'immoralité, la malhonnêteté et l'hypocrisie des politiciens.
\end{abstract}

Copy Right, IJAR, 2018,. All rights reserved.

\section{Introduction:-}

Marcel Pagnol auteur et dramatique français, né en 1895 à Aubagne, pose avec courage et netteté, les problèmes politiques en pleine lumière. Il s'amuse à se moquer des politiciens, de leur vie qui se partage entre les manipulations et le mensonge.

La politique selon lui, c'est le pouvoir,le pouvoir de dicter les autres, de profiter de chaque situation, de falsifier les choses. Comme le dit Winston Churchill: $\ll<$ Le meilleur argument contre la démocratie est un entretien de cinq minutes avec un électeur moyen.> (2) Pagnol exprime ses idées avec une liberté plus grande. Ce qui est remarquable chez Pagnol, c'est que, pour parler des politiciens, il adopte un point de vue politique. À travers Les Marchands de gloire nous pouvons bien remarquer les efforts qui ont été faits pour faire le lecture conscient des défauts du système politique.

La politique manque de transparence. Aux yeux des politiciens, ce sont leurs intérêts qui valent le plus; ce qui résulte au système corrompu. On va discuter comment Marcel Pagnol nous présente la réalité du système politique avec le maximum d'exactitude possible. Il nous montre comment les politiciens se servent des gens comme une étape au succès et les oppressent. En politique, ceux qui peuvent survivre, ce sont les puissants, les faibles sont facilement supprimés.

Il nous dit satiriquement qu'un politicien veut rouler et veut enrouler tout le monde autour de son petit doigt. Il exerce le pouvoir de domination. Ici, une citation d'André Suarès paraît juste: $<<$ En politique, la sagesse est de ne 
point répondre aux questions. L'art de ne pas se les laisser poser.>> (3) Pour son profit, il utilise une stratégie de l'utilisation de l'ensemble de ces pouvoirs.

Pour nous suggère qu'en politique, c'est le pouvoir et l'habilité qui règnent; ce qui résulte au système corrompu. Selon Nicolas Machiavel: $<<$ En politique le choix est rarement entre le bien et le mal, mais entre le pire et le moindre mal.>> (4)

Les politiciens tuent la démocratie. Ils oppressent les peuples. Comme le dit Oscar Wilde: $<<$ Démocratie: l'oppression du peuple par le peuple pour le peuple.>> (5) La démocratie; ce n'est qu'un jeu pour les politiciens. Ils vivent dans la nostalgie de leur pouvoir et l'exerce dans partout. Les mots de Louis XVI : <<C'est légal, parce que je le veux.>> (6) présente un exemple parfait ce que nous avons dit.

Un politicien n'hésite pas à écraser n'importe quoi et n'importe qui pour leur profit. L'idéalisme n'a aucune place dans la politique. Selon Louis Latzarus: $<<$ Toute revolution est commencée par des idéalistes, poursuivies par des démolisseurs et achevée par un tyran.>> (7) C'est le pouvoir qui compte.

Les politiciens se montrent sincères et honnêtes comme Marcel Pagnol a écrit dans Les Marchands de Gloire: $<<$ Mon cher, en politique, tout est comédie. Et même quand on est sincère, car ça arrive tout de même quelque fois il faut jouer la comédie de la sincérité, autrement les gens n'y croient pas.>> (8) La politique est un <<show business $>$ où le meilleur acteur gagne ou autrement ils utilisent leur pouvoir pour influencer le résultat d'élection. C'est comme un match de boxe, celui qui est plus puissant, gagne.

C'était pour acquérir le pouvoir que le drame était joué par Berlureau et Bachelet dans Les Marchands de gloire et les devoirs sont distribués parmi la foule. Les politiciens ne les permettent pas de s'exprimer ou de réagir. Comme le dit Noam Chomsky: << Le rôle du public consiste à suivre les orders, non à s'y intégrer. $>$ (9) Malheureusement les politiciens utilisent leur pouvoir dans une façon illégale ce qui résute dans la corruption et le crime.

C'est le soif du pouvoir et de la position qui préoccupre l'esprit d'un homme politique. Il utilise son pouvoir pour avoir un résultat voulu, pour falsifier les choses, pour supprimer les adversaires ou pour n'importe quoi. À l'extérieur la politique semble être fondée en un ensemble de valeurs, mais à l'intérieur tout est en désordre. L'univers politique a capté le pouvoir mais ils ne sont pas capable de gérer les problèmes des gens parce qu'ils l'ont capté pour leur profit.

Alors la politique lui donne tout le pouvoir de pratiquer tous les moyens de s'enrichir au nom du peuple qui l'a élu. Au contraire, le gouvernement ne doit pas exercer son pouvoir dans une façon illégale. Selon Henri David Thoreau: $<<$ Le gouvernement le meilleur est celui qui gouverne le moins. $>>$ (10) Pagnol nous dit que les politiciens luttent avec leurs semblables au lieu de faire des efforts pour l'amélioration de leur pays.

Pagnol aussi met en lumière la corruption politique. Sans doute nombreux d'écrivains ont parlé de la politique mais ce qui distinguee Pagnol est sa façon de metre en lumière la politique dans une façon satirique. Il nous montre comment les politiciens dupent les gens et profitent de chaque situation. Citons Jules Michelet: $<<$ La politique est l'art d'obtenir de l'argent des riches et des suffrages des pauvres, sous prétexte de les protéger les uns des autres.> (11) Presque tout le système politique est corrompu. Ce système est influencé par le trafic d'influence.

Sous le prétexte de protéger la paix et la liberté, les politiciens gardent le vrai Bonheur pour eux-mêmes. Selon Coluche: << Les gardiens de la paix, au lieu de nous la garder, ils feraient mieux de nous la foutre. > (12) Ils veulent assurer pour eux-mêmes le statut d'un roi mais ils ne veulent jamais prendre les responsabilités comme Ambrose Gwinett Bierce a dit: <<Ministre. Personne qui agit avec un grand pouvoir et une faible responsabilité.>> (13) Dans la manière que les religieux enchaînent la liberté des gens au nom de Dieu, les politiciens le font au nom des Lois.

La question de corruption politique a également mis en lumière le manqué de moralité. Pagnol nous fait voir leur rude réalité. Ils rêvent d'une victoire éclatante mais dans ce rêve l'humanité est perdu. Selon Gustave Flaubert: $<<$ La politique: quelle saleté! > (14) Pagnol nous dit comment un politicien pour promouvoir son statut et pour ses campagnes électorales ment et trahit la foi et la confiance des autres. 
Pagnol laisse son lecteur le pouvoir de former son proper opinion puisqu'il ne les jugent pas mais les présente satiriquement. Dans les mots de Costa-Gavras: $<<$ Vous ne pouvez changer la vision politique des gens avec un film, maisvous pouvez au moins engendrer une discussion politique.> (15).

Pagnol met en lumière la laideur de la politique. Les politiciens mêlent la vérité des événements avec le mensonge et la présente assez facilement. Pagnol a très bien montré dans Les marchands de gloire qu'un politicien veut faire l'argent dans n'importe quelle façon: $<<$ C'est pour gagner plus d'argent que tu veux faire ce métier. $>>$ (16)

On voit une grande similarité entre la corruption politique et la tumeur, l'un tue le pays et l'autre un être, mais les duex tuent. Mais on peut dire que la corruption politique est beaucoup plus dangereuse comme elle peut tuer tout un État dans un coup. Selon Paul Valéry: <<Toute politique tend à traiter les hommes comme des choses.» (17) Leur soif du pouvoir, de la domination et de la conquête est infinie.

Le public tombe en proie des désirs aveugles des politiciens. C'est leur art de faire le public imbécile qui les fait succéder. Pour eux, avec le pouvoir viennent la gloire et la richesse. C'est la soif d'autorité qui les surmonte. Pagnol démasque bien leurs intentions cachées.

L'habileté et surtout le tact de falsifier des choses tiennent une grande importance dans la vie politique. Les politiciens se donnent la figure d'un bienfaiteur, ils se présentent au service du public mais en réalité, c'est le contraire. Selon Léon Blum: « Toute société qui prétd assurer aux hommes la liberté, doit commencer par leur garantir l'existence.>> (18) Les principes et les norms sur lesquelles la politique est fondée sont trahis. Selon MC Solaar : < Il veut faire de la politique pour être un gangster moderne. $>>(19)$

Il n'y a pas de transparence dans la realisation de gros investissements publics. Le sale jeu de politique affecte les valeurs démocratiques. Pagnol présente satiriquement un tableau du monde politique qui parait le même pour Presque tous les pays. Les traits de satire lui servent à individualiser fortement des caractères des politiciens. Pagnol a très bien utilisé l'exagération qui est une technique de satire où l'on prend une situation et l'exagère à tel point qu'elle deviant ridicule. Selon Pagnol, Les politiciens sont impitoyables les lois sont mis au coin dans le monde politique. Comme le dit Jacques Chirac: << Le monde politique est une jungle. > (20) On veut ajouter ici que le monde politique est pire qu'une jungle.

La plus urgente nécessité pour un politicien, c'est d'acheter la position qu'il souhaite à n'importe quel prix. Il mange la société peu à peu. Henry de Montherlant a très bien dit: $<<$ La religion est la maladie honteuse de l'humanité. La politique en est le cancer.> (21) Pagnol évoque une sorte d'amertume révolté chez ses lecteurs à travers les dialogues comme: $<$ Et voilà un qui a su exploiter la mort de son fils. $>$ (22) qui frappe le lecteur au coeur. La politique est en rupture complete avec l'humanité.

Sous le masque de sincérité, les politiciens achèvent leur proper but. Comme le dit Karl Marx: $\ll$ Toute classe qui aspire à la domination doit conquérir d'abord le pouvoir politique pour représenter à son tour son intérêt propre comme étant l'intérêt général.>>(23) Les politiciens n'ont aucun sens de devoir.

C'est dans sa pièce Les Marchands de gloireque nous voyons agir Pagnol contre l'institut politique. Il exprime son mépris pour l'univers politique qui est incurablement vulgaire. Il nous montre la peinture brute de la réalité politique où il présente les politiciens comme les prisonniers du demon de l'orgueil. Citons un dialogue de sa pièce Topaze: $<<$ Madame, je suis un élu du peuple; je n'ai pas le droit de me laisser insulter.〉 (24)

Les politiciens font le public rêver de tous ce qu'ils gardent pour eux-mêmes en réalité. $<<L$ 'argent, le pouvoir, les honneurs, la jouissance, la puissance, la domination, la propriété c'est pour eux, une poignée, l'élite; pour les autres, le peuple, les petits, les sans-garde, la pauvreté, l'obéissance, le renoncement, l'impuissance, la soumission, le mal-être suffisent.> (25) Ils utilisent leur position pour flatter leurs intérêts plutôt que pour render services aux gens. C'est un diplomate qui relie habiliment la politique à l'accomplissement de ses désirs professionnels et personnels en traitant la foule comme une belle proie. Il se croit supérieur à tous.

Un politicien au lieu de garantir la liberté et l'égalité les tue: $<<$ Liberté, égalité, fraternité! Paroles vaines, funestes même, depuis qu'elles sont devenues politiques; car la politique en a fait trois mensonges.> (26) Le politicien se 
fait si puissant que personne ne peut l'enrayer. C'est à cause de tels politiciens que l'avenir de la société est abominable.

Au lieu de résoudre les problèmes, ils les créent pour en tirer le profit: $\ll$ Un diplomate est un homme qui est payé pour tenter de résoudre les difficultés qui ne se seraient jamais présentées s'il n'y avait pas eu de diplomates.〉> (27)

Pagnol a aussi parlé d'un autre vice qui existe en politique, c'est l'hypocrisie des politiciens qui paralyse le système tout entier. Il nous révèle les intentions cachées des politiciens en utilisant deux armes les plus efficacies: La satire et l'ironie. Il souligne la mesquinerie des politiciens qui encourage également le non respect des normes. Il commente sur l'immoralité de ceux qui gouvernent.

Au lieu d'être les modèles de vertus, les politiciens ne sont que les hypocrites qui veulent profiter de chaque situation et pour qui le devoir et les valeurs n'ont aucune importance. Ils savent bien l'art de bafouer les normes mais encore paraissent naïfs. Comme le dit Voltaire: $<<$ La politique est le premier des arts et le dernier des métiers. > (28) Pagnol met en lumière qu'en politique tout est comédie et si l'on veut y réussir, il faut jouer ce drame, le drame de sincérité.

Pagnol nous suggère que la politique est une sale de théâtre où nombreuses pièces d'hypocrisie, de brutalitè sont jouées sous la direction des politiciens. Ils savent exploiter chaque situation, en plus, ils savent profiter de chaque événement. Citons un dialogue de sa pièce Les Marchands de gloire: $<<$ Tragique parole qui me brise le coeur mais dont je me servirai. >> (29)

Dans sa pièce Les Marchands de gloire, un père amiable devient ou bien encore transforme à un profiteer de la mort de son fils: << Et moi-même, je me demanderais si la mort glorieuse de mon fils n'a pas servi mon ambition. > (30) Il falsifie les circonstances et cache la vérité, le retour de son fils.: $<<$ Certes, je ne puis oublier les temps heureux, où il poussait, d'une baguette légère, son cerceau, dans le jardin public de sa ville natale... Et lorsque la Patrie l'appella, je gardais comme tous les pères, l'espoir de le voir revenir...>> (31) et cette pièce se termine sur cette note qui envisage la laïdeté de la politique.

Pour les politiciens les valeurs et les normes sont complètement inutiles, obsolètes et encombrantes parce qu'ils les arrêtent de jouer le jeu qui servira leurs propres intèrês et qui résulte en souffrance de la société. Un politicien est un joueur, un acteur qui a plusieurs visages qu'il utilise de temps en temps.

Il nous semble que le but exclusif de Pagnol est de critique l'hypocrisie chez les politiciens et de prendre parti des naïfs qui souffrent à cause de ses politiciens. Pagnol a bien présenté son mépris pour l'injustice et l'hypocrisie et son amour et son respect pour l'humanité et la justice.

Les politiciens gardent le public dans une illusion et font de fausses promesses. Ils abusent la naïveté de ces gens. Selon Louis XI: << En politique, il faut donner ce qu'on n'a pas, et promettre ce qu'on ne peut pas donner. >> (32) Alors un politicien n'a pas une oreille disponible mais au contraire il n'est qu'un égoöste, un acariâtre, un profiteer.

La satire de Pagnol nous donne un message et achève son but parce que: $\ll$ Tout journal satirique, sous quelque régime que ce soit, est donc nécessairement un journal révolutionnaire, parce qu'il donne au peuple un très vif sentiment de l'infériorité de ses gouvernements. > (33) Pagnol accuse les politiciens de se montrer sincères, qui son ten réalité trop ingénieux.

En se montrant un ami, un serviteur, un politicien fait server tout le monde. Comme le dit Jean Amadou: $<<L a$ devise des mousquetaires était: $<<$ Un pour tous, tous pour un ... >> Celle des politiques est: $<<$ Un pout tous et tous pour moi...>> Ce qui n'est pas tout à fait la même chose.>>(34)

Les politiciens utilisent tous les moyens de tromperie par exemple les mots doux, la politesse, les promesses qui ne seront jamais réalisées. Selon Ambrose Bierce: <<Politesse. La plus acceptable des hypocrisie. > (35) En mettant le public dans un monde rêveur, il garde le vrai Bonheur pour lui-même. 
La satire de Pagnol n'est pas un simple divertissement mais un moyen de faire le public penser et d'agir contre les défauts du système politique. Elle est bien capable de provoquer le rire et la pensée en même temps. Pagnol nous donne l'idée que les politiciens, l'hypocrisie est une délivrance mais pour le public c'est une catastrophe. Ils jettent le public dans un état de nostalgie. Dans les mots de Jean-Paul Marat: $\ll$ Pour enchaîner les peuples, on commence par les endormir. $>(36)$ On ne peut pas se confier aux politiciens: $<<$ Amitié de cour, foi de renards et société de loups. > (37)

La politique peut tourner la tête d'un être humain, la politique peut le changer complètement. Chaque homme politique, soit du niveau bas ou haut, profite de son pouvoir et sa position et celui qui a plus de pouvoir règne les autres. Comme Jean le Rond a dit: $<<$ Il avait appris d'un politique philosophe, que les grandes places sont comme les rochers escarpés, qu'iln'y a que les aigles et les reptiles qui y parviennent.>> (38) Le domaine où ce jeu est le plus exercé, c'est le domaine d'élection.

L'attitude, le comportement d'un homme politique change d'un clin d'oeil selon les circonstances et les situations. Il ne pense qu'à ses profits. Il réserve les loisirs pour lui-même et laisse la foule faire face aux vents capricieux de la misère. Les politiciens désespèrent la foule chaque fois mais personne ne les questionne, peut-être parce qu'ils ont l'habitude et ils le prennent naturellement. Albert Camus a justement dit: $<<$ La société politique contemporaine: une machine à désespérer les hommes.>> (39)

De plus, ils trompent les gens: $<<$ L'art de la guerre, qui est l'art de détruire les hommes, comme la politique est celui de les tromper.> (40) Ils réussissent en utilisant la tromperie, l'hypocrisie comme une arme puissante. Ils s'amusent bien mais ils montrent qu'ils ont une loured tâche et la présente comme un devoir. Le dialogue, le plus cher, d'un politicien est $<<$ je suis un de vous $>>$. Il sait toutes les manières de duper les gens. La foule est traitée plus âprement, plus impitoyablement.

Comme nous avons déjà dit qu'un politicien est un aristocrate oisif et libertin qui abuse de la naïveté des gens et c'est la raison que ce petit nombre de gens gouvernent toute la nation. Selon Thomas Fuller: $<<$ La foule a beaucoup de têtes et pas de cervelle.>> (41) Les politiciens savent donner de fausses explications et d'illusions de la liberté et de la paix. Ils touchent les sentiments des gens en utilisant les mots magiques.

Cette corruption a sérieusement ménacée l'humanité. La structure du système politique n'est pas sans rappeler les crimes, la corruption et l'hypocrisie des politiciens. Pagnol fait une raillerie des politiciens et suggère assez clairement que ce sont les honneurs et la vie luxueuse qui les intéressent. Le crime, la corruption, l'hypocrisie, le clientélisme, tels sont les vices qui touchent toutes les organisations politiques sous différentes formes.

À cause de ses crimes commis par les hommes politiques, la politique est peu digne d'attirer l'attention des hommes raisonables et honnêtes. Et si par hazard, ils y entrent, elle les transforme complètement. Pagnol dénonce inlassablement les abus du pouvoir par un politicien qui fait les abus de la justice.

Pagnol présente le monde politique comme un monde dangereux où l'homme politique a ses propres règles. En politique, personne n'obéit au protocole dans l'exercice du pouvoir. La soif d'autorité surmonte les politiciens. Dans ce monde de politique, on peut voir plus de trahison que de sacrifice. Ils sont les gens qui rient quand il y de la souffrance et de la douleur dans le monde.

Pagnol démasque l'actualité politique. Pour acquérir ses ambitions, un politicien n'hésite pas à comettre les crimes. De plus, il n'hésite pas à les répéter parce que c'est son succès qui lui donne ce droit. Comme le dit John Dryden: $<<$ Seul le succès justifie le crime.>> (42) Un politicien trahit cruellement la confiance mis en lui par ses électeurs. Sous un couvert de modestie, les politiciens sont toujours engagés à jouer le jeu du pouvoir. Les désirs aveugles les détournent de leur devoir, de leur voie et ils s'engagent en pleine lutte pour acquérir le pouvoir suprême et ceux qui les opposent doivent souvent faire face aux conséquences terribles. Comme le dit Henri Queuille: << La politique ne consiste pas à faire taire les problèmes, mais à faire taire ceux qui les posent. >> (43)

\section{Notes:-}

1. Marcel PAGNOL, Topaze, CEuvres Complètes I, Théâtre, Éditions de Fallois, Paris, 1995, p. 335.

2. http://www.linternaute.com/citation/theme/95/politique.

3. http://www.linternaute.com/citation/theme/95/politique. Ibid. 
4. http://www.evene.fr/citations/theme/politique.php?

5. http://www.linternaute.com/citation/theme/95/politique.

6. http://www.evene.fr/citations/mot.php?mot=politique

7. Marcel PAGNOL, Les Marchands de gloire, Euvres Complètes I, Théâtre, Éditions de Fallois, Paris, 1995, p. 132.

8. http://www.toupie.org/citations/Pouvoir.htm

9. http://www.evene.fr/citations/theme/politique.php?page=2

10. http://www.linternaute.com/citation/theme/95/politique/.

11. http://e.fichot.free.fr/?p=citations

12. http://www.toupie.org/citations/Pouvoir.htm

13. http://www.de-la-vie.com/14-citations/politique-3/politique-3.htm

14. http://www.evene.fr/citations/mot.php?mot=politique.

15. Marcel PAGNOL, Les Marchands de gloire, CEuvres Complètes I, p. 93.

16. http://www.de-la-vie.com/14-citations/politique-4/politique-4.htm

17. http://www.proverbes-citations.com/cit5.htm.

18. http://www.linternaute.com/citation/theme/95/politique/.

19. http://www.de-la-vie.com/14-citations/politique-3/politique-3.htm Ibid.

20. Marcel PAGNOL, Les Marchands de gloire, CEuvres Complètes I, p. 83.

21. http://www.evene.fr/citations/mot.php?mot=satirique.

22. Marcel PAGNOL, Topaze, Presses pocket, Paris, 1928, p. 128.

23. http://www.toupie.org/citations/Pouvoir.htm

24. Citation de Louis Veuillot, http://www.linternaute.com/citation/theme/95/politique/

25. http://www.de-la-vie.com/14-citations/politique-3/politique-3.htm

26. http://www.linternaute.com/citation/theme/95/politique/.

27. Marcel PAGNOL, Les Marchands de gloire, CEuvres Complètes I, p.90. Ibid., p. 83. Ibid., p. 156.

28. http://www.linternaute.com/citation/theme/95/politique/.

29. Marcel PAGNOL, Notes sur le rire, Euvres Complètes I, p. 1004.

30. http://www.dicocitations.com/citation.php?mot=politique.

31. http://www.evene.fr/citations/mot.php?mot=hypocrisie.

32. http://www.evene.fr/citations/theme/paroles-hommes-politiques.php?Page=2.

33. manuel des étudies littéraires françaises, XVIIIe siècle, P.-G Castex, P.Surer, Hachette, 79, Bd St-Germain, Paris, 1966, p. 75.

34. http://www.dicocitations.com/citation.php?mot=politique

35. http://www.citationspolitiques.com/theme.php3?id_mot=98.

36. Jean le Rond d'Alembert, http://www.dicocitations.com/citation.php?mot=politique

37. http://www.intoxitation.com/categorie-humour-8-0.html.

38. http://www.citations.com/litterature-et-crime/citations-et-phrases-celebres-pour-crime-134-htm.

39. http://www.linternaute.com/citation/theme/95/politique. 